\title{
ADAPTIVE LOCAL PHASE APPROXIMATIONS AND GLOBAL UNWRAPPING
}

\author{
Jose Bioucas-Dias ${ }^{\dagger}$, Vladimir Katkovnik ${ }^{\dagger \dagger}$, Jaakko Astola ${ }^{\dagger \dagger}$, and Karen Egiazarian ${ }^{\dagger \dagger}$ \\ ${ }^{\dagger}$ Instituto de Telecomunicações and the Instituto Superior Técnico,Technical University of Lisbon, \\ 1049-001 Lisboa, Portugal. E-mail: bioucas@lx.it.pt. \\ ${ }^{\dagger}$ Signal Processing Institute, University of Technology of Tampere, \\ P. O. Box 553, Tampere, Finland. E-mail: firstname.lastname@tut.fi.
}

\begin{abstract}
The paper introduces a new modulo- $2 \pi$ phase denoising algorithm based on local polynomial approximations. The zero and first order approximations of the phase are calculated in sliding windows of varying size. The former is used for pointwise adaptive window size selection, while the latter is used for filtering the phase in the obtained windows. For unwrapping, we input the PUMA unwrapping algorithm [1] with the denoised wrapped phase obtained with the proposed approach. Simulation shows that this technique enables strong noise attenuation while preserving image details.
\end{abstract}

Index Terms - Fringe techniques, hologram, local polynomial approximation, phase unwrap, speckle interferometry.

\section{INTRODUCTION}

A variety of holographic imaging systems deal with phase measurements using coherent radiation in order to illuminate objects. The scattered return carries information on the physical and geometrical properties of objects such as shape, deformation, movement and surface's structure. The phase is a key element for both wave-front and wave-field reconstructions in 3D holographic imaging and with important applications in such areas as 3D TV, interferometric aperture radar and sonar, magnetic resonance imaging, adaptive optics, diffraction tomography, nondestructive testing of components, deformation and vibration measurements $[2,3]$.

Common to these applications is that the observations are periodic functions, defined on the interval $[-\pi, \pi)$, of the true phase, the so-called absolute phase. If the true phase is outside this interval, its observed value is wrapped into it, corresponding to an addition or subtraction of an integer number of $2 \pi$. Many approaches to absolute phase estimation follow a two-step procedure: in the first step, the wrapped phase is inferred from noisy wrapped observations; in the second step, the absolute phase is inferred from the wrapped estimates.

This work was supported by the Fundação para a Ciência e Tecnologia and the Instituto de Telecomunicações under the project IT/LA/325/2005, by the Academy of Finland, project No. 213462 (Finnish Centre of Excellence program 2006 - 2011) and by EC within FP6 (Grant 511568 with the acronym 3DTV).
The latter procedure is known as phase unwrapping. A variety of techniques have been developed for phase unwrapping $[2,4,1]$. A classical approach implements path-dependent local techniques, where a pixel-by-pixel unwrapping is confirmed by local phase congruence tests. A more recent direction formulates the phase unwrapping as an inverse problem leading to path-independent optimization techniques.

Owing to the periodic observation mechanism, phase unwrapping is known to be a hard problem that has since long fostered active research. In fact, if the magnitude of phase variation between neighboring pixels is larger than $2 \pi$, i.e., the so-called Ito condition is violated, then the inference of the $2 \pi$ multiples is an ill-posed problem. These violations may be due to spatial undersampling, discontinuities, or noise. To deal with these difficulties, some sort of a priori information shall be used. One efficient way, for both the local pathdependent and global path-independent methods, is the use of external information in the from of masks and quality maps.

The high levels of observation noise, typical of many holographic imaging modalities, introduce further difficulties in the phase reconstruction, as the phase unwrapping methods developed for noiseless data are very sensitive to noise. One of the first and natural ideas is prefiltering the noisy wrapped data and then using it for further processing, in particular for phase unwrapping. However, a phase fringe pattern is a very delicate object with crucial details easily to be damaged in prefiltering. If the noise level is small, any reasonable filtering is acceptable, However, in the heavy noisy case, the standard approaches often damage data in such a way that further unwrapping becomes impossible.

In this paper, we propose a novel filtering technique based on local polynomial approximation with varying adaptive neighborhood used in reconstruction [5]. This adaptivity is a crucial element: it assumes that for each point (pixel) there is a neighborhood where the polynomial approximation fits well the data. Both elements of this model, the coefficients of the polynomial approximation and the neighborhood size (some times and shape), are subjects of estimation. The adaptiveness of the algorithm trades bias with variance in such a way that the window size stretches in areas where the underlying true phase is smooth and shrinks otherwise, namely in the presence of discontinuities. Simulation studies show that the developed filtering is very efficient, namely in the presence of 
discontinuities. We found out, namely, that the phase unwrapping equipped with this sort of prefiltering yields very good accuracy of the phase reconstruction, quite often overcoming the state-of-the-art algorithms developed for noisy phase unwrapping.

The polynomial modeling for the phase unwrap is a popular idea. In particular, the efficiency of the local phase fitting is demonstrated in [6] for two-dimensional magnetic resonance imaging data. In the paper [7] the linear local polynomial approximation of height profiles is used for the surface reconstruction from the multifrequency InSAR data. Using the local polynomial fit in the phase tracking for the phase unwrap is proposed in the paper [8], where it is further exploited for initialization of the global optimization giving the final estimate.

The algorithm proposed in this paper is based on accurate least square fitting of the linear polynomial models. In our previous works [9], [10], this fitting is approximate using the local minimum of the local least square criterion and used for the phase unwrap in a phase tracking procedure. In this paper we are focused mainly on filtering of the wrapped phase as the prefiltering procedure for the forthcoming unwrapping.

\section{LPA PHASE-APPROXIMATION}

The standard formulation of the phase unwrapping starts from the observation model in the form $\phi=W\left(\varphi+n_{\varphi}\right)$, where $\varphi$ is the true phase and $n_{\varphi}$ is a random noise. Here $W$ is a wrapping operator transforming the noisy phase to the interval $[-\pi, \pi)$. For $n_{\varphi}=0$, there is an obvious link between the wrapped $\phi$ and non-wrapped absolute phase $\varphi, \varphi=\phi+2 \pi k$, $\phi \in[-\pi, \pi)$, where $k$ is an integer. The basic unwrapping problem is to reconstruct $\varphi(x, y), x, y \in X \subset \mathbb{Z}^{2}$, from the observations $\phi(x, y)$. There is no one-to-one relation between the wrapped and absolute phase. The approach developed in this paper is based on two independent ideas: local approximation for design of nonlinear filters (estimators) and adaptation of these filters to unknown smoothness of the varying phase. As flexible universal tools, we use local polynomial approximation (LPA) for approximation and intersection of confidence intervals $(I C I)$ for adaptation [5]. The $L P A$ is applied for filter design using a polynomial fit in a sliding window. The window size as well as the order of the polynomial define a desirable filter. The window size is considered as a varying adaptation parameter of the filter. The $I C I$ is an adaptation scheme that searches for a largest local window size where the variance and the bias of the estimates are balanced. It is shown that the $I C I$ adaptive $L P A$ yields nearly optimal minimum mean squared error estimates.

The observation model assumed in this paper is of the form

$$
z=A \exp (j \varphi)+n
$$

where $n=n_{I}+j n_{Q}$ is complex-valued zero-mean circular white noise of variance $2 \sigma^{2}$ (i.e., $n_{I}$ and $n_{Q}$ are zero-mean independent Gaussian random variables with variance $\sigma^{2}$ ).
Assume that in some neighborhood of the point $(x, y)$ the phase $\varphi(x, y)$ can be represented in the form

$$
\tilde{\varphi}\left(x_{s}, y_{s} \mid \mathbf{c}\right)=p^{T}\left(x_{s}, y_{s}\right) \mathbf{c}
$$

where $p=\left(p_{1}, p_{2}, p_{3}\right)$ is a vector of the first order polynomials $p_{1}=1, p_{2}=x, p_{2}=y$, and $\mathbf{c}=\left(c_{1}, c_{2}, c_{3}\right)^{T}$ is a vector of unknown parameters. To infer this vector, we compute

$$
\hat{\mathbf{c}}=\arg \min _{\mathbf{c}} L_{h}(\mathbf{c})
$$

where $L_{h}(\mathbf{c})$ is a measure of the data misfit defined as

$$
\begin{aligned}
& L_{h}(\mathbf{c})= \\
& \quad \frac{1}{2} \sum_{s} w_{h, s} \mid z_{\phi}\left(x+x_{s}, y+y_{s}\right)-\exp \left(\left.j \tilde{\varphi}\left(x_{s}, y_{s} \mid \mathbf{c}\right)\right|^{2}=\right. \\
& \quad \sum_{s} w_{h, s}\left\{1-\cos \left[\phi\left(x+x_{s}, y+y_{s}\right)-\tilde{\varphi}\left(x_{s}, y_{s} \mid \mathbf{c}\right)\right]\right\},
\end{aligned}
$$

where $z_{\phi} \equiv z /|z|=e^{j \phi}$. It can be verified by routine calculations that for the considered linear phase model the optimal solution $\hat{\mathbf{c}}$ is of the form

$$
\begin{aligned}
\left(\hat{c}_{2}, \hat{c}_{3}\right) & =\arg \max _{c_{2}, c_{3}}\left|F_{h}\left(c_{2}, c_{3}\right)\right| \\
\hat{c}_{1} & =\operatorname{angle} F_{h}\left(\hat{c}_{2}, \hat{c}_{3}\right),
\end{aligned}
$$

where $F_{h}\left(c_{2}, c_{3}\right)$ is the windowed Fourier transform of the normalized data $z_{\phi}=e^{j \phi}$ with the arguments $c_{2}, c_{3}$, thus meaning the frequency components of the exponential signal $e^{j \phi}$ at spatial frequency $\left(c_{2}, c_{3}\right)$; i.e.,

$$
F_{h}\left(c_{2}, c_{3}\right)=\sum_{s} w_{h, s} z_{\phi}\left(x+x_{s}, y+y_{s}\right) e^{-j\left(c_{2} x_{s}+c_{3} y_{s}\right)} .
$$

Then, the phase estimate $\hat{c}_{1}$ is the argument (angle) of the complex-valued Fourier transform $F_{h}$ calculated at the point where the maximum value of the Fourier spectrum is achieved. The formulas (4) show that the standard Fourier transform can be used for implementation of the proposed algorithm.

According to the model (2), the local polynomial model for the neighborhood of the pixel $(x, y)$ is $\hat{\varphi}\left(x+x_{s}, y+y_{s}\right) \equiv$ $p^{T}\left(x_{s}, y_{s}\right) \hat{\mathbf{c}}(x, y)$. In particular, we have

$$
\hat{\varphi}(x, y)=\hat{\mathbf{c}}_{1}(x, y) .
$$

In general, the estimate $\hat{\mathbf{c}}(x, y)$ depends of the coordinates $(x, y)$ and the window size $h$. We wish to emphasize the nonparametric nature of the introduced estimator. Indeed, we start from the parametric linear on $x$ and $y$ model in (2) and could expect that the approximation (estimate) is also linear on $x$ and $y$. However, the fit is used in the polynomial approximation (2) only for one "central" point $x_{s}=y_{s}=0$. The result of this point-wise approximation is that the parametric estimate (2) becomes nonparametric, with $\hat{\varphi}(x, y)$ depending in a nonlinear way on $x$ and $y$ [5]. All ideas of the standard $L P A$ concerning the window $w$ (shape, anisotropy, directionality, etc.), the scaling $h$ (scalar, multivariate), estimation of 
the signal and derivatives are naturally valid in this nonparametric pointwise estimation.

As final remark on the LPA approach, we note that a zeroorder approximation of the phase would lead to the estimate

$$
\tilde{\varphi}(x, y)=\tilde{c}_{1}(x, y)=\operatorname{angle}\left(F_{h}(0,0)\right)
$$

\section{ESTIMATE ACCURACY AND ADAPTATION}

Given a two-dimensional window function $w$ and a window size (scale) parameter $h>0$, we define the scaled window $w_{h, s} \equiv w\left(x_{s} / h, y_{s} / h\right)$, centered at $(x, y)$. In particular, if we take the square uniform window $w=1$ for $|x| \leq 1,|y| \leq 1$ and $w=0$ otherwise, we have $w_{h}=1$ for $|x| \leq h,|y| \leq h$ and $w_{h}=0$ otherwise. A smaller or larger $h$ narrows or widens the window $w_{h}$, respectively.

It is shown in Proposition 2 of [9] that, asymptotically for small noise level and symmetrical windows, the phase estimate $\hat{\varphi}(x, y)=\hat{\mathbf{c}}_{1}(x, y)(5)$ is unbiased with the variance

$$
\sigma_{h}^{2}=\frac{\sigma^{2}}{A^{2}} \frac{\sum_{s} w_{h, s}^{2}}{\left(\sum_{s} w_{h, s}\right)^{2}} .
$$

This result is used for the adaptive selection of the window size $h$. Let $H$ be a set of the ordered window sizes $H=\left\{h_{1}<h_{2}<\ldots<h_{J}\right\}$ and $\hat{\varphi}_{h}(x, y)$, for $h \in H$, the respective phase estimates. A statistic known as the intersection of confidence interval $(I C I)$ rule is exploited in order to select the best window size. Given the estimates $\hat{\varphi}_{h}(x, y)$ and the respective variance, for $h \in H$, the confidence intervals of these estimates are defined as

$$
Q_{h}=\left\{\hat{\varphi}_{h}-\Gamma \cdot \sigma_{h}, \hat{\varphi}_{h}+\Gamma \cdot \sigma_{h}\right\} .
$$

where $\Gamma>0$ is a parameter of the algorithm and $\sigma_{h}$ is calculated according to (7).

The $I C I$ rule defines the adaptive window size, denoted by $h^{+}$, as the largest $h \in H$ for which the estimate $\hat{\varphi}_{h}$ does not differ "significantly" from the estimates corresponding to the smaller window sizes. In order to identify this adaptive $h^{+}$, the successive intersection of the confidence intervals $Q_{h}$ is considered starting from $Q_{h_{1}}$ and $Q_{h_{2}}$. Specifically, the pairwise intersection of the intervals $Q_{h_{j}}, 1 \leq h_{j} \leq h_{i}$, is considered with increasing $h_{i}$. Let $h^{+}$be the largest of those $h_{i}$ for which the intervals $Q_{h_{j}}, 1 \leq h_{j} \leq h_{i}$, have a point in common. This $h^{+}$defines the adaptive window size and the adaptive estimate as $\hat{\varphi}_{h^{+}}$.

For the varying pointwise adaptive estimation, these calculations are produced for all points (pixels). In implementation, the $I C I$ algorithm is used when the estimates for all points $(x, y)$ are already calculated for all $h$. Then the algorithm works as a selector of the proper window size estimate for each point from a given set of the estimates for all window sizes ([5], Chapter 6).

Parameter $\Gamma$ in (8) controls the bias-variance balance in the estimate. Decreasing $\Gamma$ means a shift of this balance in favor of the bias, as smaller $\Gamma$ results in smaller bias of the
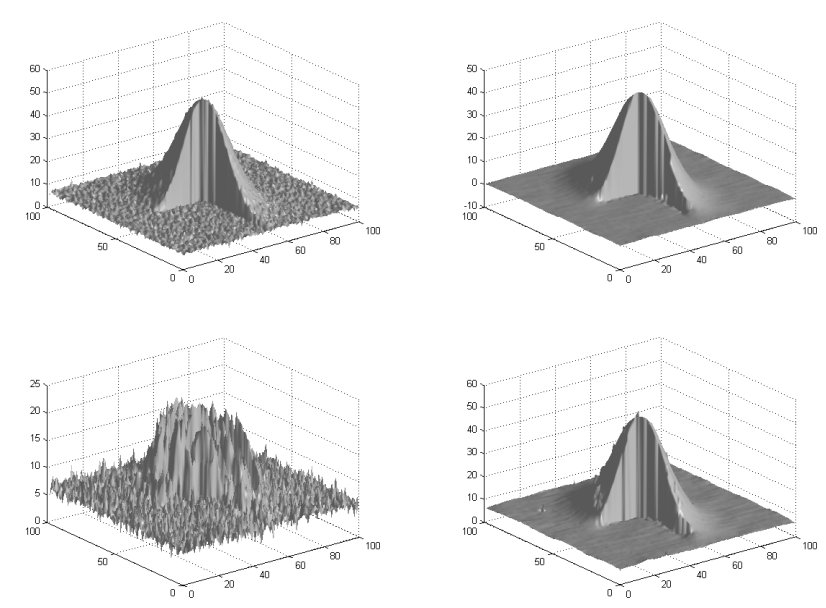

Fig. 1. From left to right: $P U M A$ reconstruction from the noisy data and from prefiltered PEARLS data, first line $\sigma=$ 0.5 and second line $\sigma=0.75$.

estimate. On the contrary, increasing $\Gamma$ means a shift in favor of the variance, as larger $\Gamma$ results in smaller variance of the estimate but possible larger bias.

\section{PEARLS ALGORITHM}

We name the proposed algorithm PEARLS for Phase Estimation using Adaptive Regularization based on Local Smoothing. The pseudo-code of this algorithm is as follows:

1: For every pixel $(x, y)$ and $h \in H$ :

a: calculate the zero-order phase estimate according to the formula (6), i.e.,

$$
\tilde{\varphi}_{h}(x, y)=\operatorname{angle}\left(\sum_{s} w_{h, s} z_{\phi}\left(x+x_{s}, y+y_{s}\right)\right)
$$

b: apply the ICI rule to the estimates $\tilde{\varphi}_{h}(x, y)$ for the selection of the best window size $h^{+}(x, y)$;

c: using the formulas (4) with $h=h^{+}(x, y)$, calculate $\hat{\varphi}_{h^{+}}$, the first-order phase estimates with adaptive window size ;

\section{End for ;}

2: unwrap phase $\hat{\varphi}_{h^{+}}$using one of the procedures developed for noise-less data, for example, the PUMA algorithm [1].

We have used zero-order estimates in the $I C I$ rule. There are at least two reasons for this: First, it makes calculations much faster. Second, the $I C I$ rule works better with zeroorder estimates than with first order ones as, the former are essentially less noisy. 


\section{SIMULATION EXPERIMENTS}

In this section, we present two experiments illustrating the PEARLS competitiveness. For the phase unwrap of the filtered wrapped phase we use the $P U M A$ algorithm [1], which is able to work with discontinuities. In what follows, the $L P A$ is exploited with the uniform square windows $w_{h}$ defined on the integer symmetric grid $\{(x, y):|x|,|y| \leq h\}$. The $I C I$ parameter was set to $\Gamma=2.0$ and the window sizes $H=$ $\{1,2,3,4\}$. The noisy observations were generated according to (1), with $A=1$, on the square grid with integer arguments $x, y,-49 \leq x, y \leq 50$. The absolute phase is defined by the formula $\varphi(x, y)=A_{\varphi} \exp \left(-x^{2} /\left(2 \sigma_{x}^{2}\right)-y^{2} /\left(2 \sigma_{y}^{2}\right)\right)$ with $\sigma_{x}=10, \sigma_{y}=15$, and $A_{\varphi}=14 \pi$. The the maximum value of $\varphi$ is $14 \pi$ and the maximum values of the first differences about 2.5 radians. Which such high phase differences, even a noise of small variance leads to a difficult unwrapping problem, due to many phase differences larger the $\pi$.

Table 1 shows the RMSE values obtained with the PEARLS algorithm, as a function of the observation noise standard deviation $\sigma$. For comparison purposes, we have also computed the $R M S E$ obtained with the PhaseLa [10] and $Z \pi M$ [4] algorithms, which implement filtering plus unwrapping.

Table 1. RMSE for PEARLS, Phase La and $Z \pi M$ algorithms.

\begin{tabular}{|l|c|c|c|c|c|}
\hline Algorithm $\backslash \sigma$ & 0.75 & 0.5 & 0.25 & 0.05 & 0.01 \\
\hline PEARLS & 0.34 & 0.15 & 0.09 & 0.05 & 0.03 \\
\hline PhaseLa & - & 0.18 & 0.11 & 0.04 & 0.03 \\
\hline$Z \pi M$ & 1.39 & 0.21 & 0.12 & 0.19 & 0.08 \\
\hline
\end{tabular}

The unwrapping based on the PEARLS prefiltering yields consistently comparable or better performance than PhaseLa and $Z \pi M$ algorithms. The advantage increases for large values of $\sigma$, corresponding to the more challenging scenarios.

The $3 D$ imaging in Figure 1 illustrates performance of the algorithm for a discontinuous phase. In the first line we can see the reconstructions obtained by the PUMA algorithm with no prefiltering and with prefiltering. The prefiltering obviously improves the reconstruction for the smooth areas where the larger values of the window sizes are used in PEARLS algorithm. A distribution of the adaptive window sizes for this case can be seen in Figure 2. These results are shown for $\sigma=0.5$. For a larger noise level of $\sigma=0.75$, the unwrapping result without prefiltering is catastrophic, whereas with prefiltering the reconstruction is quite acceptable, despite the high noise level present in the observed data. PhaseLa and $Z \pi M$ were not compared in this experiment because they are conceived to blindly deal with discontinuities.

These results confirm conclusions obtained from multiple test experiments. The novel algorithm demonstrates very strong filtering properties enabling the posterior application of unwrapping.
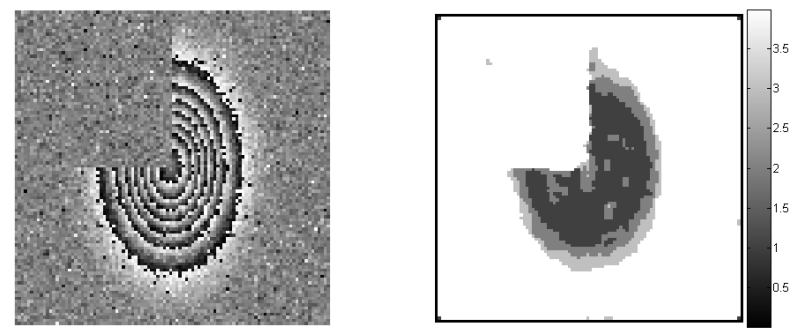

Fig. 2. Noise data and adaptive ICI window sizes, $\sigma=0.5$.

\section{REFERENCES}

[1] J. Dias and G. Valadão, "Phase unwrapping via graph cuts," IEEE Trans Image Processing, vol. 16, no. 3, pp. 684-697, 2007. vol. 25, no. 1, 2006.

[2] D. C. Ghiglia and M. D. Pritt. Two-Dimensional Phase Unwrapping: Theory, Algorithms, and Software. New York: Wiley, 1998.

[3] Th.Kreis, Handbook of Holographic Interferometry (Optical and Digital Methods), Wiley-VCH Gmbh\&Co.KGaA, Weinheim, 2005.

[4] J. Dias and J. Leitao, "The $Z \pi M$ algorithm: a method for interferometric image reconstruction in SAR/SAS," IEEE Trans Image Processing, vol. 11, $\mathrm{n}^{0}$ 4, pp. 408-422, 2002.

[5] V. Katkovnik, K. Egiazarian, J. Astola, Local Approximation Techniques in Signal and Image Processing. SPIE PRESS, Bellingham, Washington, 2006.

[6] Zhi-Pei Liang, "A model-based method for phase unwrapping," IEEE Transactions on Medical Imaging, vol. 15, no. 6, pp. 893-897, 1996.

[7] V. Pascazio, and G. Schirinzi, "Multifrequency InSAR height reconstruction through maximum likelihood estimation of local planes parameters, IEEE Transactions on Image Processing,, vol. 11, no. 12, pp. 1478-1087, 2002.

[8] M. Servin, J. L. Marroquin, D. Malacara, and F. J. Cuevas, Phase unwrapping with a regularized phase-tracking system," Applied Optics, vol. 37, no.10, pp. 1917-1923, 1998.

[9] V. Katkovnik, J. Astola and K. Egiazarian, "Phase local approximation (PhaseLa) technique for phase unwrap from noisy data," IEEE Trans on Image Processing, 2008 (excepted).

[10] V. Katkovnik, J. Astola, K. Egiazarian, "Noisy phase unwrap for holographic techniques: adaptive local polynomial approximations," Proceedings 3D TV Conference, Kos, Greece, 2007. 